\title{
Activity-Based Therapies To Promote Forelimb Use after a Cervical Spinal Cord Injury
}

\author{
Haining Dai, ${ }^{1, \star}$ Linda MacArthur, ${ }^{1, \star}$ Marietta McAtee, ${ }^{1}$ Nicole Hockenbury, ${ }^{1}$ J. Lille Tidwell, ${ }^{1}$ Brian McHugh, ${ }^{1}$ \\ Kevin Mansfield, ${ }^{1}$ Tom Finn, ${ }^{1}$ Frank P.T. Hamers, ${ }^{2}$ and Barbara S. Bregman ${ }^{1}$
}

\begin{abstract}
Significant interest exists in strategies for improving forelimb function following spinal cord injury. We investigated the effect of enriched housing combined with skilled training on the recovery of skilled and automatic forelimb function after a cervical spinal cord injury in adult rats. All animals were pretrained in skilled reaching, gridwalk crossing, and overground locomotion. Some received a cervical over-hemisection lesion at C4-5, interrupting the right side of the spinal cord and dorsal columns bilaterally, and were housed in standard housing alone or enriched environments with daily training. A subset of animals received rolipram to promote neuronal plasticity. Animals were tested weekly for 4 weeks to measure reaching, errors on the gridwalk, locomotion, and vertical exploration. Biotinylated dextran amine was injected into the cortex to label the corticospinal tract. Enriched environments/daily training significantly increased the number and success of left reaches compared to standard housing. Animals also made fewer errors on the gridwalk, a measure of coordinated forelimb function. However, there were no significant improvements in forelimb use during vertical exploration or locomotion. Likewise, rolipram did not improve any of the behaviors tested. Both enriched housing and rolipram increased plasticity of the corticospinal tract rostral to the lesion. These studies indicate that skilled training after a cervical spinal cord injury improves recovery of skilled forelimb use (reaching) and coordinated limb function (gridwalk) but does not improve automatic forelimb function (locomotion and vertical exploration). These studies suggest that rehabilitating forelimb function after spinal cord injury will require separate strategies for descending and segmental pathways.
\end{abstract}

Key words: anatomical plasticity; enriched environment; functional recovery; rehabilitation; skilled forelimb use

\section{Introduction}

$\mathbf{M}$ AMMALIAN CENTRAL NERVOUS SYSTEM (CNS) neurons generally fail to regenerate after injury, resulting in a limited recovery of function. Previous studies document the benefits of activity on plasticity and functional recovery after CNS injury. Rodents housed in enriched environments, designed to enhance motor activity and increase social interactions, exhibit increased sensory, cognitive, and motor function relative to those housed in standard cages and show increased cortical weight, dendritic branching, hippocampal neurogenesis, and synaptic strength (van Praag et al., 2005; Nithianantharajah and Hannan, 2006). Enriched environments promote improved locomotor function after spinal cord injury (Lankhorst et al., 2001; Van Meeteren et al., 2003;
Hutchinson et al., 2004; Engesser-Cesar et al., 2005), increased motor function after experimental stroke (Ohlsson and Johansson, 1995; Johansson, 1996; Biernaskie and Corbett, 2001; Risedal et al., 2002; Komitova et al., 2005) and, combined with increased sensory stimulation and specific motor training, reversal of some motor deficits following brain injury (Biernaskie and Corbett 2001; Maegele et al., 2005).

However, these interventions tend to favor the recovery of locomotion and automatic behavior; the recovery of skilled function after spinal cord injury remains problematic. Several studies suggest that functional recovery after CNS injury is dependent on the specific post-injury training paradigm. Intensive reach training improved recovery of reaching after a cervical spinal cord injury but impaired performance on an untrained ladder walking task (Girgis et al., 2007). Likewise,

\footnotetext{
${ }^{1}$ Department of Neuroscience, Georgetown University Medical Center, Washington, DC.

${ }^{2}$ Rudolf Magnus Institute of Neuroscience, Department of Physical Medicine and Rehabilitation, Ultrecht, The Netherlands.

*These authors contributed equally to this work.
} 
running on a motorized wheel after stroke, prior to reach training, facilitated recovery of forelimb motor function in the staircase reaching test but had no effect on recovery of postural support (vertical exploration) or ladder walking (Ploughman et al., 2007). Running alone is reported to be of limited benefit in the recovery of forelimb movement (Risedal et al., 2002; Marin et al., 2003). Therefore, understanding the principles by which experience shapes both skilled and unskilled recovery is critical for developing strategies to rehabilitate the injured central nervous system.

In this study we examined the recovery of skilled reaching, coordinated movement on a gridwalk, vertical exploration, and overground locomotion after a cervical spinal cord injury and rehabilitation. Forelimb function is dependent on both local circuitry as well as supraspinal input. The relative dependence of forelimb function on supraspinal input depends on whether the function being measured is automatic or skilled. For example, automatic limb movements, such as forelimb use during vertical exploration or locomotion, require some corticospinal input, but are largely controlled at the level of the spinal cord and brainstem (Metz et al., 1998; Muir and Whishaw 1999; Juvin et al., 2005; Piecharka et al., 2005). In contrast, skilled movements away from the body, such as directed forepaw retrieval (reaching), are dependent upon cervical propriospinal neurons, which receive input from corticospinal, rubrospinal, raphespinal, reticulospinal, and tectospinal axons, which then project to brachial motorneurons (Alstermark and Kummel, 1986; Alstermark et al., 1987, 1990; Piecharka et al., 2005). Segmental and supraspinal descending pathways integrate with propriospinal neurons and must be coordinated with postural support to free the forelimb for use (Castro, 1972; Alstermark and Kummel 1986; Whishaw et al., 1986, 1998; Alstermark et al., 1990; Weidner et al., 2001). Thus, forelimb use during locomotion and posture relies on different neural circuitry than during forelimb reaching.

We examined the effect of increased activity (enriched environments) combined with forelimb training in skilled reaching and traversing an elevated gridwalk on the recovery of skilled and automatic forelimb function after a cervical spinal cord injury. Adult rats were simultaneously pretrained to reach for food pellets, a measure of distal forelimb impairments (Whishaw et al., 1986), and to traverse an elevated gridwalk, a measure of coordinated forelimb placement and sensorimotor function analogous to the ladder crossing test (Barth et al., 1990). They were further trained to traverse a glass runway to measure forelimb function during locomotion (CatWalk) (Hamers et al., 2001), although no additional locomotor training was provided. Some animals received a right-sided cervical over-hemisection lesion of the spinal cord at C4-5, interrupting the right side of the spinal cord and the dorsal columns bilaterally. This lesion produces severe longterm deficits in both forelimbs, with more deficits on the right side than the left, and with some impairment in the right hindlimb (Lynskey et al., 2006). Two days after lesion, rats were housed in either enriched environments with daily training in reaching, gridwalk, and CatWalk, or in standard housing, and were handled but not trained. A subset of animals housed in both conditions received rolipram, a phophodiesterase inhibitor associated with increased anatomical plasticity and recovery of motor function (Krause and Kuhne, 1988). Recovery of left and right reaching, sensorimotor func- tion on the gridwalk, locomotion on the CatWalk, and vertical exploration were measured weekly for 4 weeks after injury. Corticospinal tract plasticity was measured within the spinal cord after completion of the behavior studies.

\section{Materials and Methods}

\section{Animals}

Adult female Sprague-Dawley rats (approximately 6 weeks old at the start of the study) were used (Zivic Laboratories, Zelienopole, PA). Rats were housed in the Georgetown University Division of Comparative Medicine and had unlimited access to food and water throughout the duration of the experiment with the exception of training for the behavior measurements. A total of 54 rats were used for the behavior studies. Two rats were excluded due to autotomy of their left side. An additional 18 rats were used to measure cAMP levels within the spinal cord. All protocols were approved by the Georgetown University Animal Care and Use Committee.

\section{Study design}

To ensure objectivity, the investigators were blind to the specific treatment of each rat. Four behavior tests were used to measure forelimb function. Reaching for food pellets was used to measure skilled distal and proximal forelimb function and skilled use of the digits. Gridwalk crossing was used to measure coordinated motor and sensory motor function in all four limbs. Vertical exploration in a cylinder was used to measure automatic forelimb function and shoulder use. Automatic locomotion was assessed using the CatWalk gait analysis system.

Animals were food deprived for up to 48 hours before pretraining for the behavior tests. Animals were then maintained on scheduled feeding to limit the rate of growth to $2-5 \mathrm{~g}$ per day to motivate them to train for food rewards. Rats were pretrained daily for 6-7 days for reaching, gridwalk, and CatWalk (Fig. 1A). On days 8-9, rats were filmed (time 0) and unrestricted food was restored day 9 after testing. On days $11-12$, rats underwent surgical over-hemisection lesions of the spinal cord at C4-5 (HX). At the time of surgery, a subset of animals that were lesioned also received either rolipram $(0.4 \mu \mathrm{mole} / \mathrm{kg} / \mathrm{h}$; Sigma-Aldrich, St. Louis, $\mathrm{MO})$ in vehicle (saline/15\% DMSO; HX + Rp), or vehicle alone (HX + Veh), administered subcutaneously for 10 days at $10 \mu \mathrm{L} / \mathrm{h}$ by miniosmotic 2ML1pump (Alzet, Cupertino, CA).

Two days after surgery animals were separated into either standard housing (standard environment) or enriched environment cages designed to enhance physical activity and social stimulation. They were subsequently food deprived for retraining and then food restricted. Four days after surgery, animals housed in enriched environment cages received daily reach training (20 min), gridwalk training (10 $\mathrm{min})$, and CatWalk training (4 runs). Animals housed in standard cages were handled and their shoulders gently rotated to maintain flexibility, but they did not receive training. At 1 week after surgery, all animals were filmed and assessed for reaching, gridwalk, vertical exploration, and CatWalk. During the second and subsequent weeks, rats housed in enriched environment cages underwent daily training on reaching, gridwalk, and CatWalk while animals housed in standard cages were handled but not trained on the behavior tests. All ani- 


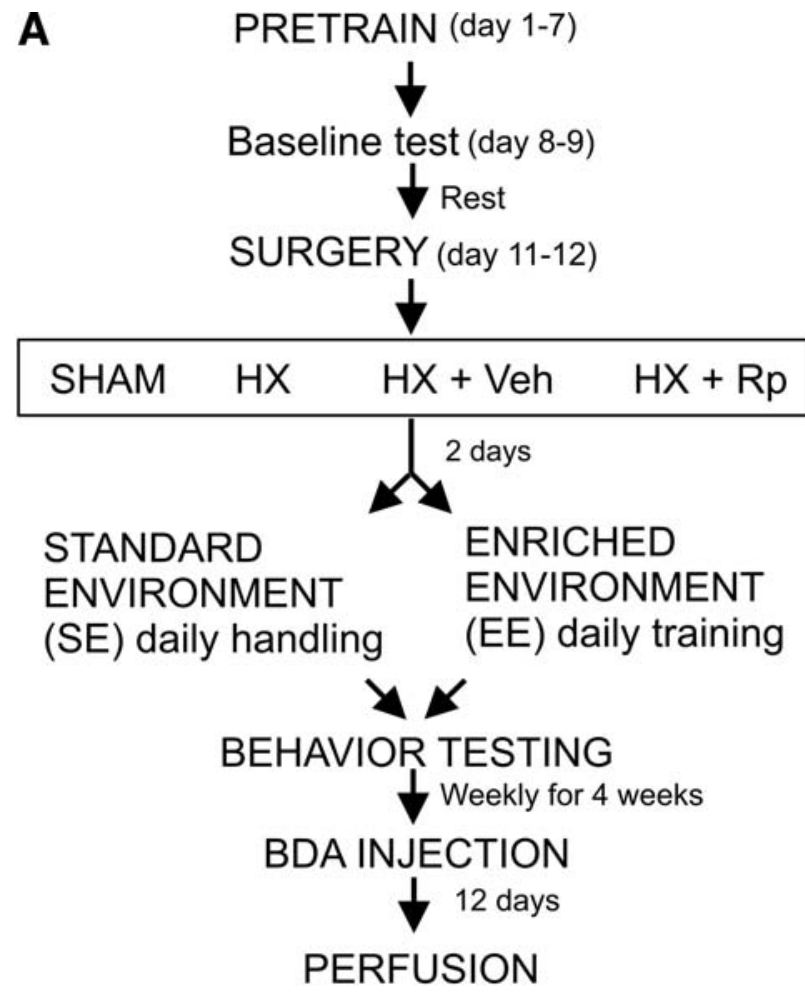

B

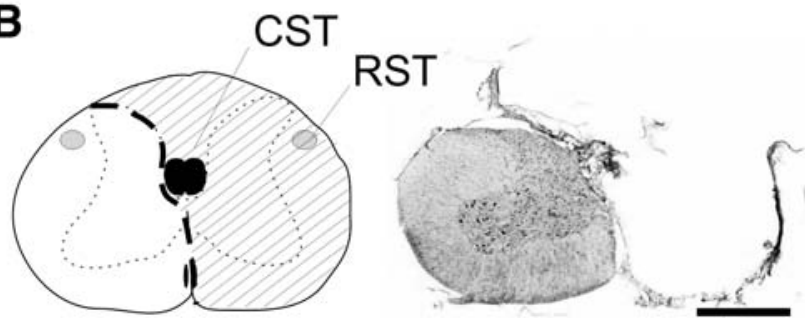

FIG. 1. Experimental design. (A) Animals were pretrained in reaching, gridwalk, and CatWalk and then underwent cervical over-hemisection lesions (HX) of the right spinal cord at C4-5. A subset of animals with lesions received rolipram (Rp) or vehicle (Veh). Animals were divided into those housed in standard environments with daily handling (SE) or enriched environments with daily training (EE) in reaching, gridwalk, and CatWalk. Animals were tested weekly for 4 weeks. BDA was injected into the motor cortex of all of the animals at the conclusion of the behavior study and animals were perfused for histology 12 days later. (B) A transverse section of the spinal cord at the lesion site. The left schematic shows the location of the corticospinal tract (CST) and the rubrospinal tract (RST). The cross-hatched region shows the area removed by the over-hemisection. The right panel shows a sample transverse section from the lesion site stained with cresyl violet. scale bar $=1 \mathrm{~mm}$.

mals were tested and filmed at 1,2,3, and 4 weeks post-injury on all four behavior tests. At the end of the behavioral analysis (4 weeks after surgery), biotinylated dextran amine (BDA) was injected bilaterally at multiple sites throughout the sensorimotor cortex for anterograde tracing of the corticospinal tract. Twelve days later, animals were perfused and the spinal cord was dissected and processed for immunohistochemistry.

\section{Surgical over-hemisection lesion at C4-5}

The surgical techniques have been described previously (Bregman et al., 1993; Lynskey et al., 2006). Briefly, adult rats were anesthetized with $4 \%$ chloral hydrate $(0.01 \mathrm{cc} / \mathrm{g})$ intraperitoneally. A laminectomy was done and iridectomy scissors were used to create spinal cord over-hemisection lesions at C4-5. This lesion destroys the right side of the cord plus the dorsal columns bilaterally. Sham operates received a laminectomy only without a spinal cord lesion. At the end of the study, all lesion sites were reconstructed from serial cresyl violet sections. Animals with incomplete lesions were excluded from the study $(\mathrm{n}=4)$. A cross-section from lesioned spinal cord stained with cresyl violet is shown in Figure 1B. Lesions were verified to be at the C4-5 level and to encompass the entire right-sided white matter and the dorsal funiculi bilaterally. This ensured complete unilateral lesion of the rubrospinal pathway and bilateral lesion of the dorsal corticospinal pathway.

\section{Experimental housing conditions}

For environmental enrichment, animals were mixed and randomly divided into control and enriched groups. Standard environment rats were housed in standard cages $(20 \times 42 \times 21 \mathrm{~cm})$ with two to three animals per cage whereas enriched environment rats were housed in larger plexiglass cages $(90 \times 60 \times 60 \mathrm{~cm})$ with seven to nine animals per cage. Enriched environment cages contained rodent toys of various shapes, a shelf, hollow tubes, a ramp, and a running wheel. Water and food were provided on opposite sides of the cage to encourage activity. Toys were rearranged or changed twice a week and rats were kept in the enriched environment for the duration of the experiment.

\section{Rehabilitative training and behavioral testing}

The reaching, gridwalk, and rearing tests were filmed using a Canon video camcorder (GL2) at a shutter speed of 1/500 s. Frame-by-frame analysis was done with Adobe Premier Pro 2.0 software (San Jose, CA). The CatWalk test was filmed with a Pulnix (TM72EX) CCD camera with a Matrix Vision frame grabber (PCimage-SRGB) using CatWalk analysis software, version 6.12 (Noldus Information Technology, Leesburg, VA).

Skilled target reaching. Distal forelimb impairment was assessed with a pellet retrieval test (Whishaw et al., 1986). In this test, rats learn to reach for and grasp food pellets. We used a vertical version of the test to assess the use of the right and left forelimbs, as previously described with minor modifications (Diener and Bregman, 1998; Lynskey et al., 2006). This version of the test requires postural support and allows free choice of the limb. The clear acrylic box consisted of twelve $2.5 \times 2.5 \times 2.5 \mathrm{~cm}$ chambers ( 4 rows high, 3 rows across) with three pellets placed in each chamber. For training, rats were pretrained for $15 \mathrm{~min}$ on day $1,10 \mathrm{~min}$ on day $2,8 \mathrm{~min}$ on days $3-5$, and $5 \mathrm{~min} /$ day on days $6-7$, and filmed on days 8-9. Food pellets were positioned toward the front of each chamber and moved back as training progressed. For testing, animals were filmed at 1, 2, 3, and 4 weeks after over-hemisection for $5 \mathrm{~min}$. The number of left and right reaches were recorded as well as the number of pellets successfully grabbed (successful reaches). 
Gridwalk. Coordinated forelimb placement was assessed using a grid runway, as previously described (Barth et al., 1990; Kunkel-Bagden et al., 1993). Animals traverse an elevated grid runway $(27 \mathrm{~cm} \times 180 \mathrm{~cm})$ with $50 \times 50 \mathrm{~mm}$ holes for a food reward at the end of the platform. Each test session consisted of four runs per day on two days, and the average number of limb placement errors, or foot faults (when the foot slips through the grid opening) per run, was noted.

Vertical exploration. Forelimb asymmetry was assessed during vertical exploration, as previously described (Schallert et al., 2000). This test encourages upright postural exploring. Animals were placed in a clear, open-top cylinder measuring $26.5 \mathrm{~cm}$ in height and $17.5 \mathrm{~cm}$ in diameter. When in this cylinder, animals spontaneously rear onto their hindlimbs and vertically explore the walls while actively using the palmar surface of their forelimbs for support. Animals were filmed for 3 min per trial while in a cylinder. Analysis consisted of recording the number of rears, the number of times each forelimb actively contacted the wall of the cylinder during a rear, and the quality of forelimb use during wall contact (dorsal versus palmar).

CatWalk gait analysis. Overground locomotion was analyzed using an automated quantitative analysis system termed the CatWalk (Hamers et al., 2001, 2006). The CatWalk gait analysis system (v.6.1) measures, among other attributes, interlimb coordination, swing duration, stride length, base of support, and relative pressure during locomotion. Prior to surgery, animals were pretrained to cross a glass runway measuring $120 \mathrm{~cm}$ long and $10 \mathrm{~cm}$ wide for a food reward (pellets from BioServe, Frenchtown, NJ) in a darkened room. After 5 days of training, three crossings per day for 2 days were analyzed for each animal (six runs total). Post-injury testing was conducted at 1, 2, 3, and 4 weeks after injury. Footprints were identified manually and the CatWalk program calculated average forelimb and hindlimb base of support, stride length, stand time, and swing time.

\section{Anatomical tract tracing, tissue preparation, and immunohistochemistry}

For corticospinal tract tracing, both sensorimotor cortices were exposed bilaterally by removing two areas of the skull from $2.5 \mathrm{~mm}$ anterior to bregma to $2.5 \mathrm{~mm}$ posterior to breg$\mathrm{ma}$ and from 1 to $4 \mathrm{~mm}$ lateral to the midline using a Dremel microtool drill (Dremel, Renovo, PA). Once the cortices were visualized, $5 \mu \mathrm{L}$ of $10 \%$ biotinylated dextan amine (BDA, 10,000; Molecular Probes, Eugene, OR) in 0.1 M phosphate buffer was injected in $0.3-0.4 \mu \mathrm{L}$ aliquots $(\sim 15-16$ injections) over $7 \mathrm{~min}$ throughout each forelimb sensorimotor cortex ( $2 \mathrm{~mm}$ anterior and posterior of bregma 1.5-3.5 $\mathrm{mm}$ lateral to the midline. Injections were $1-1.5 \mathrm{~mm}$ deep).

Twelve days later, animals were anesthetized and perfused with $4 \%$ paraformaldehyde in $0.1 \mathrm{M}$ phosphate buffer. Serial cryosections of spinal cord $(20 \mu \mathrm{m})$ were cut in a transverse plane rostral to lesion for visualization of BDA labeled corticospinal tract fibers. Longitudinal sections were cut through the lesion site to visualize the extent of the lesion. Sections were cut in a 1:6 series and thaw-mounted onto SuperFrost Plus slides (Fisher, Pittsburgh, PA). Slides from each set were used for immunohistochemistry, and adjacent slides were stained with cresyl violet to confirm the accuracy of the lesion.
BDA staining was performed to visualize corticospinal projections $8 \mathrm{~mm}$ rostral to lesion as described previously (Reiner et al., 2000) with modifications (Lynskey et al., 2006).

\section{Quantification of area occupied by labeled fibers}

All sections were captured under identical conditions with respect to magnification, illumination, and exposure time to minimize variability. Three to four sections per animal were analyzed. Quantitative analysis was performed on coded sections by individuals unaware of the experimental treatment groups. The fiber area of the BDA-labeled corticospinal tract and the dorsal column area were calculated using SigmaScan Software (Jandel Scientific, San Rafael, CA). Averages for each animal were normalized to sham controls housed in standard environments and expressed as a percent of control. Data was analyzed with GraphPad Prizm software (GraphPad Software, San Diego, CA).

\section{cAMP levels in the spinal cord}

To measure the levels of cAMP in the spinal cord after rolipram treatment, a separate set of animals received a cervical over-hemisection lesion. Two weeks later, either rolipram $(0.4 \mu$ mole $/ \mathrm{kg} / \mathrm{h}$; Sigma-Aldrich) in vehicle (saline $/ 15 \%$ DMSO) or vehicle alone was administered for 5 days at $10 \mu \mathrm{L} / \mathrm{h}$ via mini-osmotic pump (Alzet, Cupertino, CA). At the end of rolipram treatment, animals were perfused with heparinized saline and spinal cord tissue was dissected and stored at $-20^{\circ} \mathrm{C}$. Frozen tissue was homogenized in 10 volumes of $0.1 \mathrm{~N} \mathrm{HCl}$, centrifuged at $10,000 \mathrm{rpm}$ for $10 \mathrm{~min}$ at $4^{\circ} \mathrm{C}$. Extracts were stored at $-20^{\circ} \mathrm{C}$. Protein assays were performed with the BCA method (Pierce, Rockford, IL). Protein homogenates from the rostral cervical spinal cord at $\mathrm{C} 1$ were normalized for protein content and analyzed for cAMP levels using a low pH Elisa assay (R\&D Systems, Minneapolis, MN).

\section{Statistics}

The entire behavior and anatomy study was repeated once and the data were combined. The results from reaching were analyzed with a three-way analysis of variance (ANOVA) followed by a two-way ANOVA with Bonferonni's post-hoc analysis to compare the effect of enriched environments with daily training, rolipram, or their combination on recovery of function. The results for gridwalk, vertical exploration, and CatWalk were analyzed using a two-way ANOVA with Bonferonni's post-hoc analysis as above. The quantitative results of the BDA and cAMP analyses were analyzed using a two-way ANOVA and a one-way ANOVA with Tukey's post-hoc analysis. For the reaching analysis, a repeated measures ANOVA was used for between group comparisons (enriched environments versus standard housing, and rolipram versus vehicle or no treatment) over 1-4 weeks. All data are presented as the mean \pm standard error. $P$ values of $<0.05$ were considered statistically significant.

\section{Results}

\section{Enriched environments with daily training improves recovery of left reaching}

Animals were assessed for skilled reaching at 1, 2, 3, and 4 weeks after injury and treatment. This reaching paradigm 
requires rats to reach into a shelf to retrieve pellets (Fig. 2A). A reach is counted if the left or right paw crosses into the box. The reach is considered successful if a pellet is retrieved (Fig. 2B). After a right-sided C4-5 cervical over-hemisection, animals spontaneously recovered some ability to reach with their left forelimb (Fig. 2C). A three-way ANOVA was performed to compare the effects of housing, treatment group, and limb. There was a significant effect of treatment vs. limb
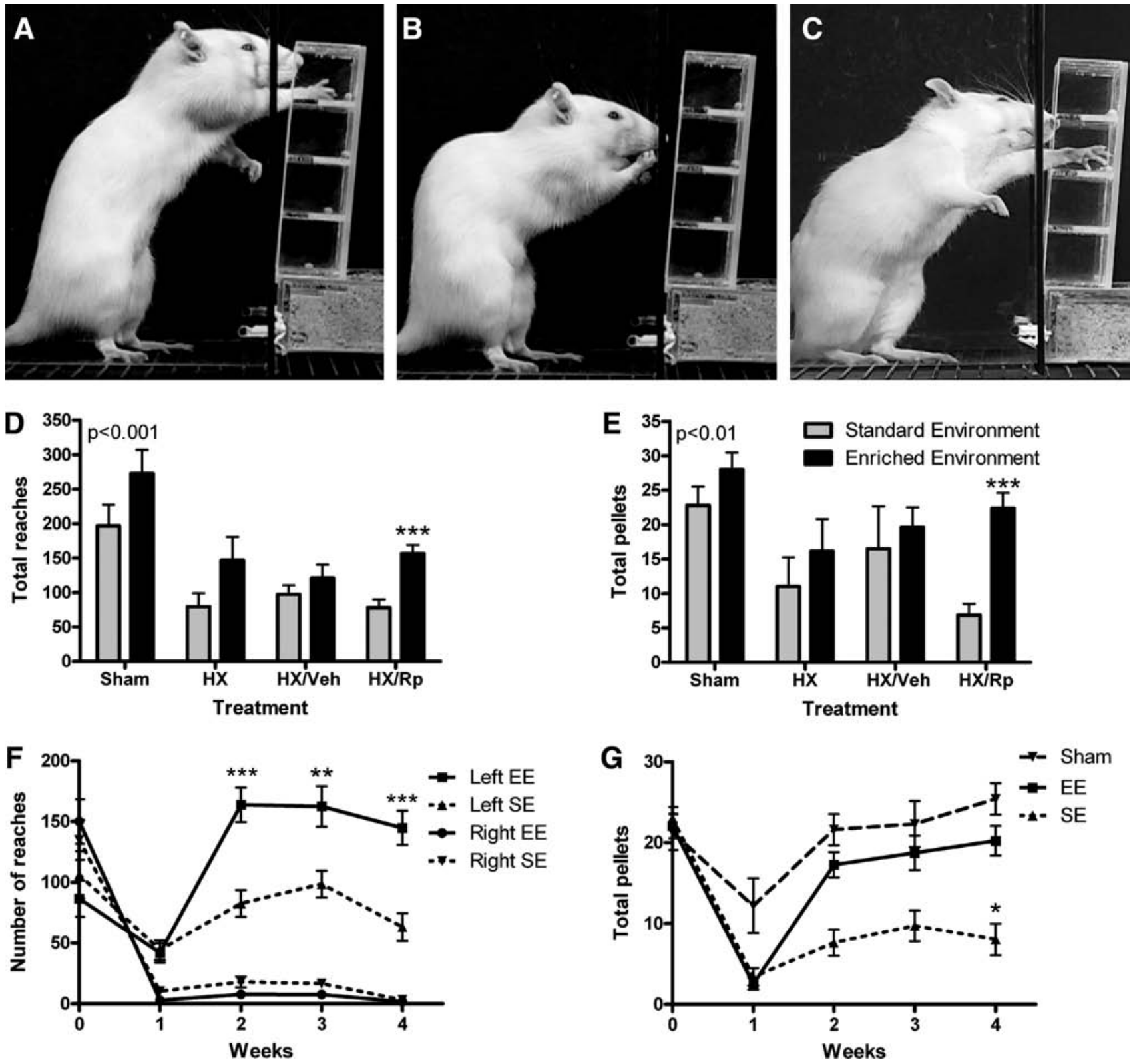

FIG. 2. Enriched environments with daily training improves left reaching. (A) The total number of times animals reached into an acrylic chamber with their forepaw to attempt to retrieve a food pellet was measured during 5 min sessions at weeks $1,2,3$, and 4 after injury with a maximum number of pellets $=36$. (B) The total number of times animals successfully retrieved and ate a pellet was measured as successful reaches. (C) After a right-sided, cervical over-hemisection, animals spontaneously recovered some ability to reach with their left forepaw. A two-way ANOVA was used to measure the effect of enriched environments with daily training (enriched environments) or rolipram (Rp) on the total number of reaches (D) or total pellets retrieved (E) 4 weeks after an over-hemisection. Values represent the mean \pm SEM. Standard environment: sham, $\mathrm{n}=5$; $\mathrm{HX}$, $\mathrm{n}=5 ; \mathrm{HX} /$ Veh, $\mathrm{n}=4 ; \mathrm{HX} / \mathrm{Rp}, \mathrm{n}=8$. Enriched environment: sham, $\mathrm{n}=5 ; \mathrm{HX}, \mathrm{n}=6$; HX/Veh, $\mathrm{n}=5 ; \mathrm{HX} / \mathrm{Rp}, \mathrm{n}=11$. (F) Recovery of left and right reaching was measured over 4 weeks. A repeated measures ANOVA was used to compare animals housed in enriched environments with daily training (EE, $n=16-22$, solid line) with those housed in standard housing (SE, $\mathrm{n}=17-21$, dashed line). Left, left forelimb; right, right forelimb. (G) A repeated measures ANOVA was used to assess the effect of daily training on the number of pellets retrieved. Animals housed in enriched environments with daily training retrieved significantly more pellets compared to those housed in standard housing or sham-operated animals. Sham, sham-operated rats; HX, cervical over-hemisection of the spinal cord; Veh, vehicle; Rp, rolipram. ${ }^{*} p<0.05,{ }^{* *} p<0.01,{ }^{* * *} p<0.001$. 
Right forelimb
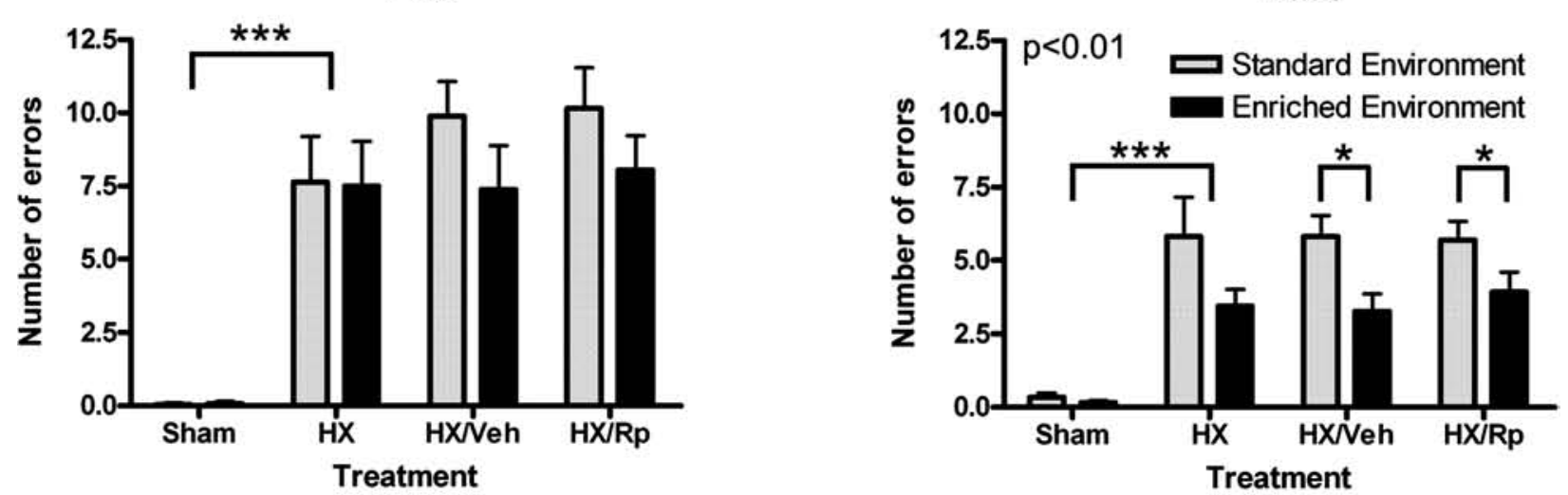

Right hindlimb
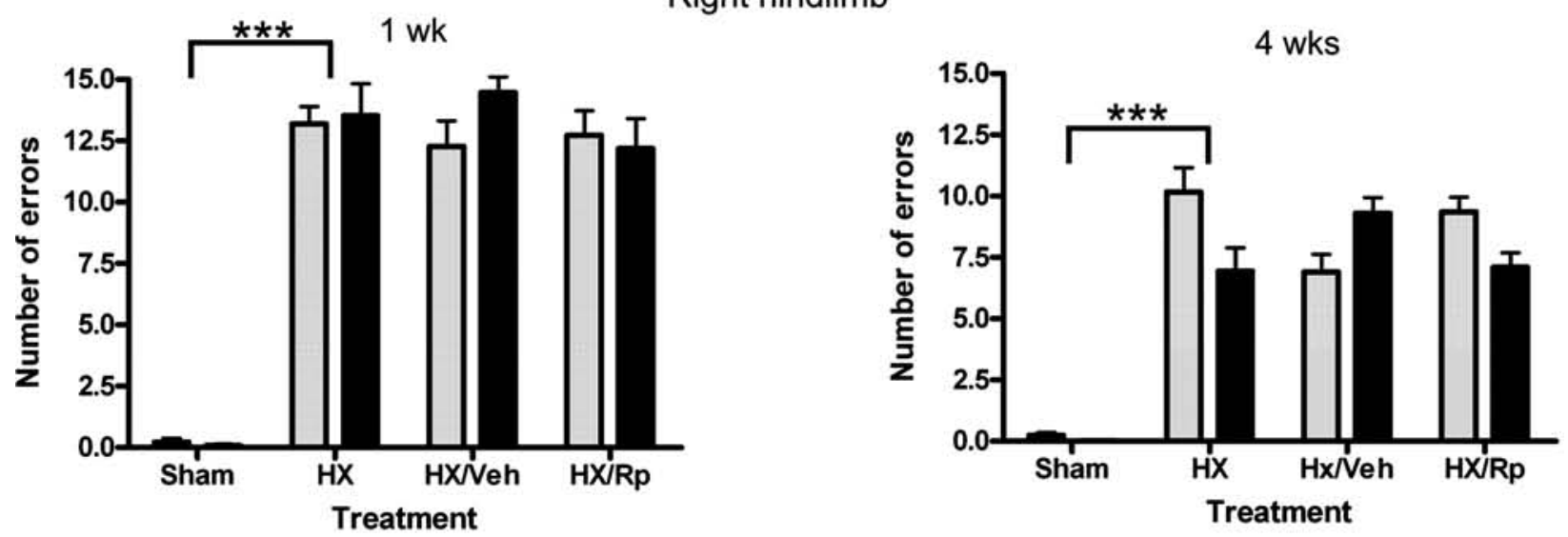

Left hindlimb

1 wk

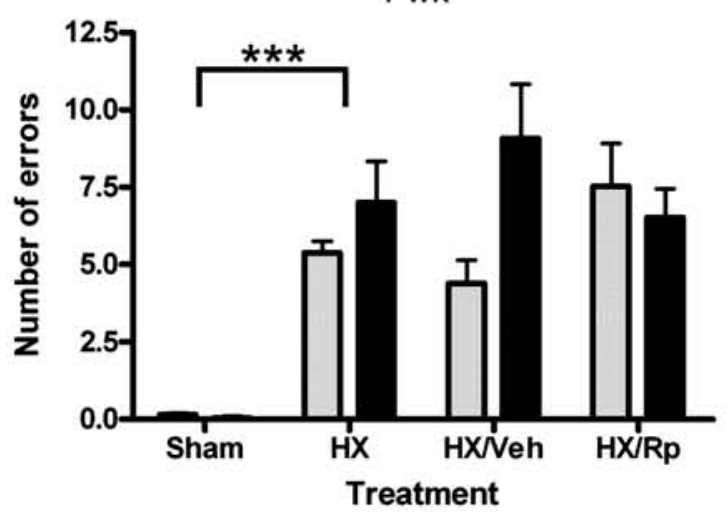

$1 \mathrm{wk}$

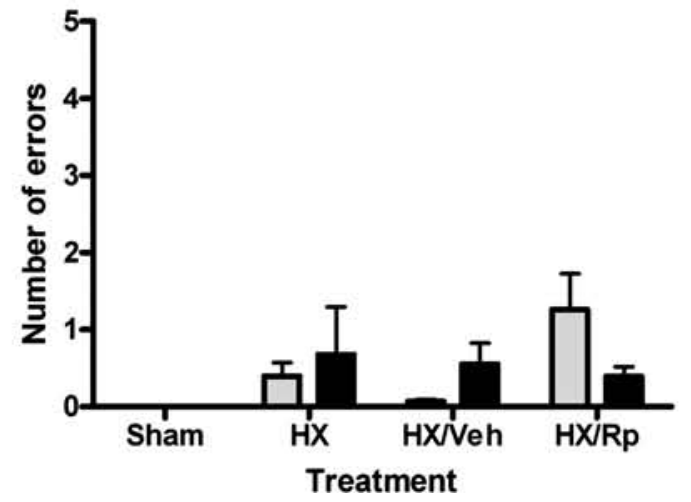

4 wks

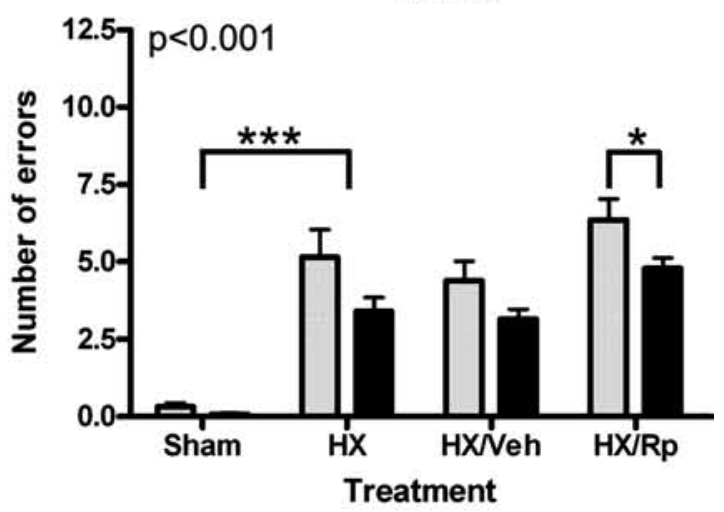

4 wks

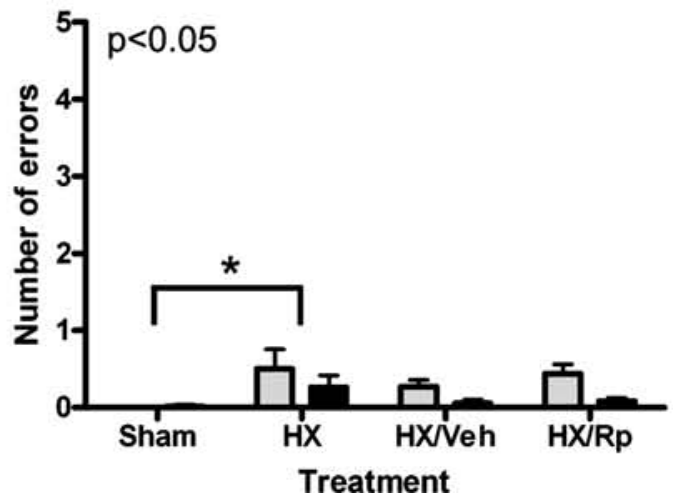


$(p<0.001)$ and housing vs. limb $(p<0.025)$. This occurred because right reaching did not recover. Therefore, two-way ANOVAs were used to compare the effect of enriched environments with daily training and rolipram on the total number of reaches (Fig. 2D) and the total number of successful pellet retrievals (Fig. 2E). There was a significant decrease in total reaches and pellets retrieved after surgical overhemisection (sham vs. HX, $p<0.0001$ and $p<0.01$, respectively). Rats housed in enriched environments with daily training had a significant increase in the number of reaches compared to animals housed in standard environments $(p<0.001$; Fig. 2D) and a significant increase in the number of pellets retrieved ( $p=0.01$; Fig. 2E). This improvement persisted through 4 weeks of testing (the time point shown). There was no significant effect of rolipram on the number of reaches or pellets retrieved, and there was no significant effect of combining enriched environments and daily training with rolipram on the total number of reaches or pellets retrieved at any time point.

Recovery of reaching was due to recovery of left reaching and not right reaching. The number of left reaches significantly increased after housing in enriched environments with daily training (Fig. 2F; left EE) by 2 weeks after injury compared to housing in standard environments (Fig. 2F; left SE). Right reaching did not recover in animals housed in either condition (Fig. 2F; right EE and right SE). Animals housed in enriched environments with daily training retrieved significantly more pellets compared to those housed in standard environments (Fig. 2G, EE vs. SE) at 4 weeks after injury.

\section{Enriched environments with daily training improves coordinated forelimb function}

Rats were assessed weekly for the number of foot faults made by each limb on a gridwalk after injury and treatment. This test is analogous to the ladder crossing test and requires both coordinated motor and sensory function to avoid errors. Before surgery, rats averaged less than one error per limb per run on the gridwalk. At 1 week after a right over-hemisection (HX), there was a significant increase in the number of errors made in all limbs, with the most errors occurring with the right forelimb, right hindlimb, and left hindlimb $(p<0.0001$, sham vs. HX; Fig. 3). Rats showed little impairment in the left forelimb. By 4 weeks after injury, enriched environments with daily training significantly reduced the number of errors made by the right forelimb $(p<0.01)$, left hindlimb $(p<0.001)$, and left forelimb $(p<0.05)$, but did not reduce the number of errors made by the right hindlimb, which had the most errors after injury. There was no significant reduction in errors made in animals treated with rolipram, and there was no significant interaction between enriched environments and daily training and rolipram.

\section{Enriched environments with daily training does not improve forelimb function during vertical exploration}

Vertical exploration in a cylinder was used to test automatic forelimb function. Normal animals placed in a test cylinder rear up on their hindlimbs and place the palmar surface of their forepaws against the cylinder wall (Fig. 4A). After a cervical over-hemisection injury, rats maintained postural support and were able to rear up on a glass cylinder, but primarily used their left forelimbs for support while the right limb remained at their side (Fig. 4B). There was a significant decrease in contacts with the right forelimb (Fig. 4D; sham vs. HX, $p<0.0001$ ) but no significant decrease in the number of contacts made with the left side (Fig. 4C). There was no significant increase in right forelimb contacts after enriched environments with daily training, rolipram, or their combination.

\section{Enriched environments with daily training slightly improves hindlimb but not forelimb function during locomotion on the CatWalk}

Animals were assessed for recovery of normal locomotion by using the CatWalk gait analysis system. This program reconstructs the gait pattern for each limb and calculates multiple measures for each step cycle, including base of support (the distance between the hindlimbs or forelimbs), stride length (the distance between paw contacts for each step cycle), standtime (the contact time for each paw during each step cycle), and swingtime (the time the paw spends in the air in between contacts) (Fig. 5A). After a cervical over-hemisection, there was an increase in the hindlimb base of support (Fig. 5B, sham vs. HX, standard environment), indicative of reduced trunk stability. Rats housed with enriched environments and daily training showed a significant decrease in hindlimb base of support to preinjury distances $(p<0.01)$. There was no significant effect of rolipram treatment. The forelimb base of support increased after injury in both housing environments (Fig. 5C, sham vs. HX). However, there was greater variability between animals and groups and no significant effect of either enriched housing with daily training or rolipram on the distance between forelimbs. Four weeks after injury, animals developed an abnormal gait characterized by a reduced stride length in all four limbs, an increased standtime in three out of four limbs, and a decreased swingtime in the left forelimb (Table 1). None of these measures was significantly affected by either enriched environments with daily training or rolipram.

\section{Corticospinal tract plasticity rostral to lesion increases after enriched environments with daily training}

The corticospinal tract is required for normal forelimb function. We used anterograde tracing with BDA to examine

FIG. 3. Enriched environments with daily training improves coordinated limb function on a grid runway. Rats were assessed weekly for the number of errors made while crossing a grid runway. The average number of limb placement errors made for each limb over six to eight runs on the grid was obtained for each animal. A two-way ANOVA was used to measure the effect of enriched environment with daily training or rolipram (Rp) on the total number of errors. Data shown are from 1 week and 4 weeks after injury and treatment. Values represent the mean \pm SEM. Standard environment: sham, $\mathrm{n}=5 ; \mathrm{HX}$, $\mathrm{n}=6$; HX/Veh, $\mathrm{n}=6$; HX/Rp, $\mathrm{n}=9$. Enriched environment: sham, $\mathrm{n}=5 ; \mathrm{HX}, \mathrm{n}=5 ; \mathrm{HX} / \mathrm{Veh}, \mathrm{n}=5 ; \mathrm{HX} / \mathrm{Rp}, \mathrm{n}=9$. Sham, sham-operated rats; HX, cervical over-hemisection of the spinal cord; Veh, vehicle; Rp, rolipram. ${ }^{*} p<0.05,{ }^{* *} p<0.01$, $* * * p<0.001$ 

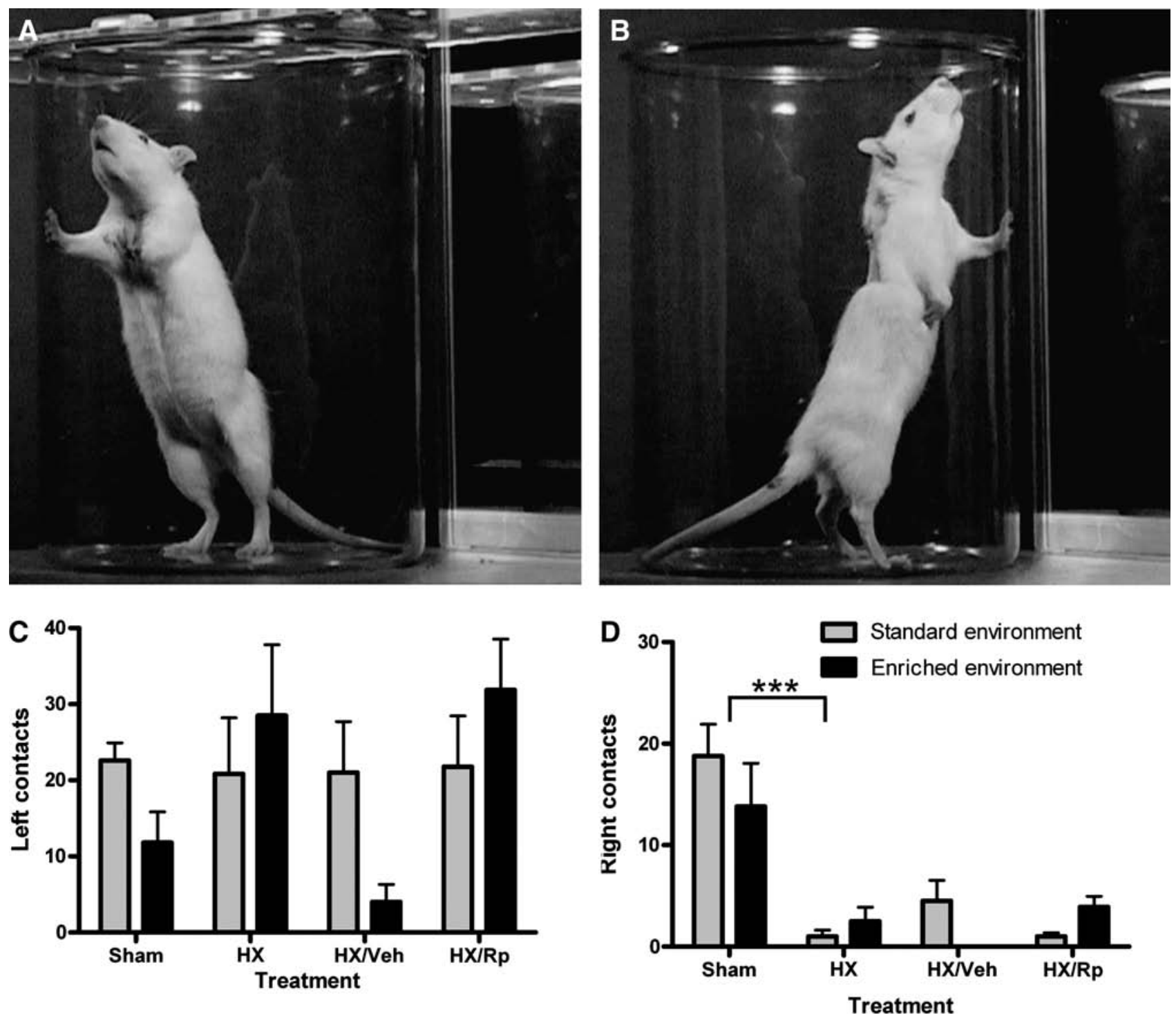

FIG. 4. Enriched environments with daily training did not improve forelimb function during vertical exploration. The number of times that animals used their right or left forelimbs while vertically exploring a glass cylinder was measured during 3-min trials. (A) Normal uninjured rats were able to raise both forelimbs during vertical exploration. (B) After a right-sided cervical over-hemisection, animals lost the ability to use their right forelimb in this test. A two-way ANOVA was used to measure the effect of enriched housing and daily training or rolipram on the total number of left contacts (C) and right contacts (D). Data shown are from 4 weeks after injury and treatment. Values represent the mean $\pm \mathrm{SEM}$. Standard environment: sham, $\mathrm{n}=5 ; \mathrm{HX}, \mathrm{n}=6 ; \mathrm{HX} /$ Veh, $\mathrm{n}=6 ; \mathrm{HX} / \mathrm{Rp}, \mathrm{n}=9$. Enriched environment: sham, $\mathrm{n}=5 ; \mathrm{HX}, \mathrm{n}=6 ; \mathrm{HX} / \mathrm{Veh}, \mathrm{n}=5 ; \mathrm{HX} / \mathrm{Rp}, \mathrm{n}=10$. Sham, sham-operated rats; HX, cervical over-hemisection of the spinal cord; Veh, vehicle; Rp, rolipram. ${ }^{* * *} p<0.001$.

plasticity of the corticospinal tract rostral to lesion. Because this study used a large cervical lesion, we were worried about possible brainstem changes in BDA transport that would interfere with using the pyramidal tract to normalize labeling, as is typically done. Therefore, total BDA positive fiber area within the dorsal columns $8 \mathrm{~mm}$ rostral to lesion, which correspond to the C1-2 spinal cord, was analyzed. The lesion site itself was not used because both dorsal columns were ablated at or proximal to the lesion. Investigators were blind to the experimental groups. While this way of measuring BDA labeling in the spinal cord was less sensitive than with normalization to the pyramidal tract, it ensured that the data were not biased by treatment effects.
BDA labeling of the corticospinal tract in uninjured control animals was intense in the dorsal columns rostral to injury (Fig. 6A). After over-hemisection, there was a dramatic decrease in labeled dorsal column fibers (Fig. 6B and C) to less than $5 \%$ of uninjured controls ( $\mathrm{HX}$ standard environment vs. sham enriched environment, $100 \%$ of control, $p<0.001)$. After over-hemisection, animals housed in enriched environments with daily training or those that received rolipram had increased BDA fiber density compared to HX alone. However, while this trend was there for all treatment groups, it did not reach significance by a two-way analysis of variance. When similar groups were combined and the density of BDA labeling was compared using a student's $t$-test, the 
effect was significant (enriched housing vs. standard housing, $p<0.05$; rolipram vs. vehicle $/ \mathrm{HX}, p<0.01$ ). After lesion, all injured animals had significantly smaller dorsal columns compared to sham-operated animals housed in either standard or enriched environments (Fig. 6D). However, there
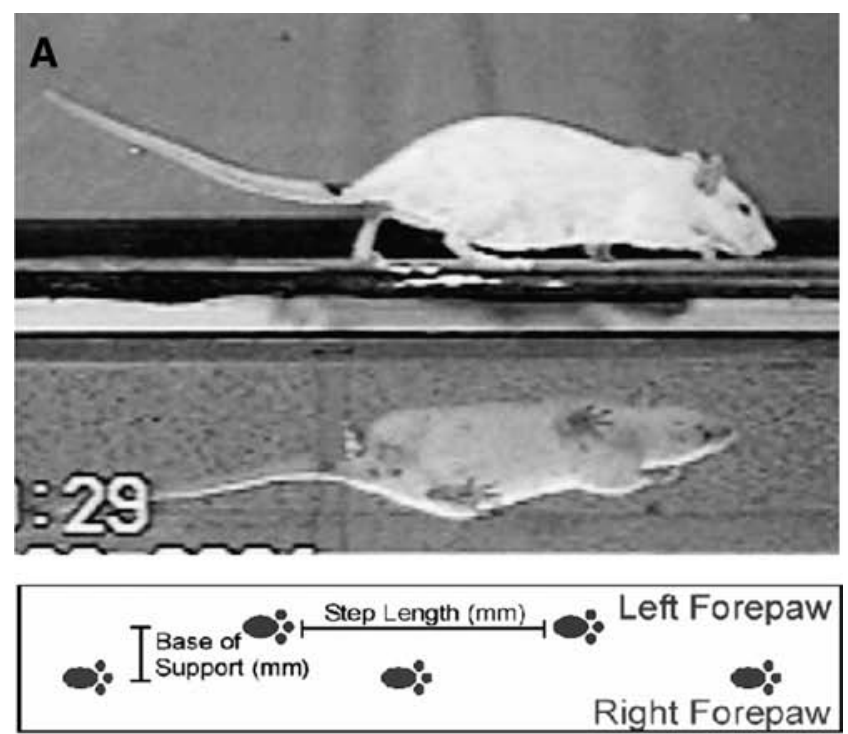

B

Standard environment Enriched environment
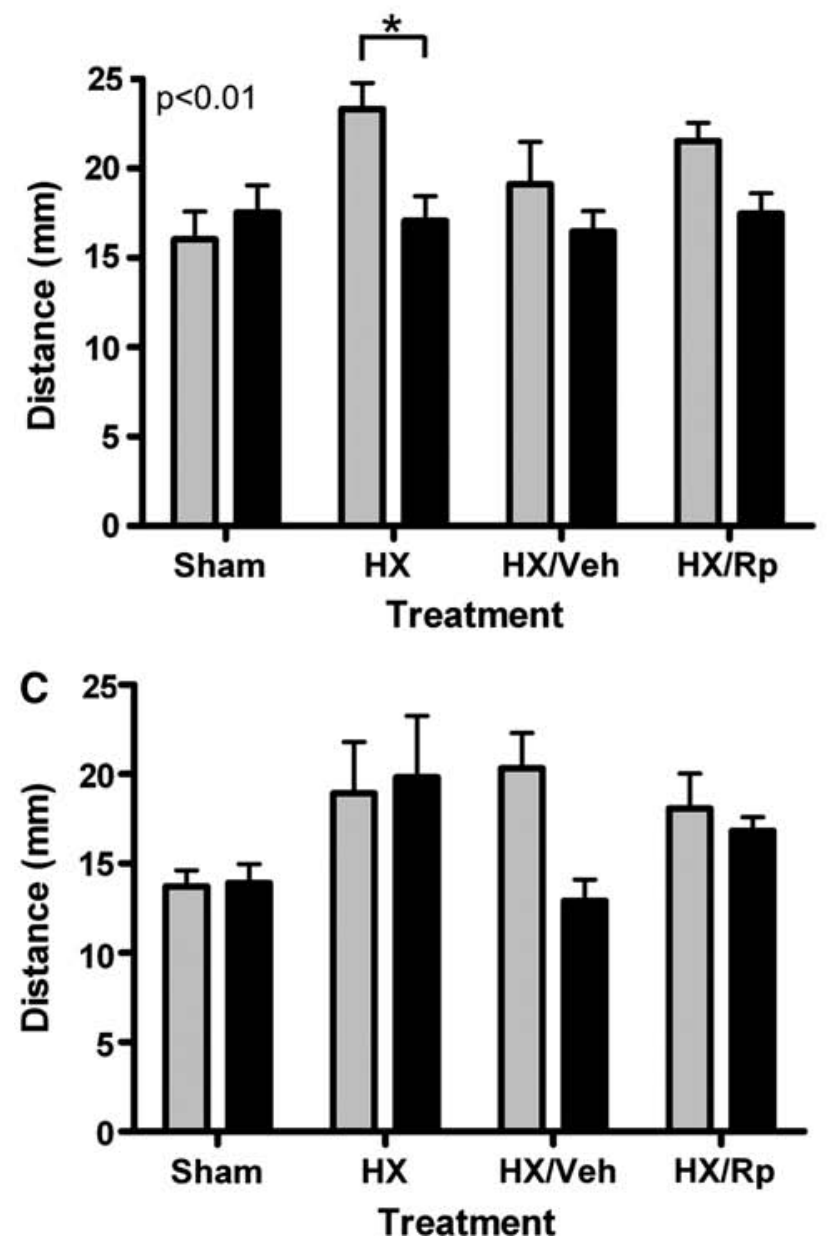

Table 1. Summary of CatWalk Gait Analysis

\begin{tabular}{lcccc}
\hline & $\begin{array}{c}\text { Left } \\
\text { Forelimb }\end{array}$ & $\begin{array}{c}\text { Left } \\
\text { hindlimb }\end{array}$ & $\begin{array}{c}\text { Right } \\
\text { forelimb }\end{array}$ & $\begin{array}{c}\text { Right } \\
\text { hindlimb }\end{array}$ \\
\hline Stride length & $\downarrow^{* * *}$ & $\downarrow^{* * *}$ & $\downarrow^{* * *}$ & $\downarrow^{* *}$ \\
Standtime & $\uparrow^{* *}$ & $\uparrow^{* *}$ & $\leftrightarrow$ & $\uparrow^{* *}$ \\
Swingtime & $\downarrow^{* * *}$ & $\leftrightarrow$ & $\leftrightarrow$ & $\leftrightarrow$ \\
\hline
\end{tabular}

Week 4 after injury. Down arrow, decrease; up arrow, increase; side arrows, no change.

${ }^{* *} p<0.01 ;{ }^{* * *} p<0.001$.

was no difference in dorsal column area between treatment groups.

In a separate control experiment, spinal cord was dissected from animals after rolipram administration, and cAMP levels at spinal cord level $\mathrm{C} 1$ were analyzed using an ELISA assay to confirm activity and delivery of the drug (Fig. 6E). After overhemisection, delivery of rolipram significantly increased cAMP content in the spinal cord compared to spinal cord from normal $(p<0.05)$, rolipram-treated $(p<0.01)$, or injured only $(p<0.05)$ (Fig. 6E). Neither hemisection alone (HX) nor rolipram delivery to uninjured spinal cord $(\mathrm{Rp})$ affected cAMP content. Saline with DMSO (HX + Veh) slightly increased the cAMP content of the cord, although this was not significantly different from normal controls. These data suggest that enriched environments, daily training, and rolipram promote plasticity of the spinal cord rostral to lesion after injury.

\section{Discussion}

In this study we show that enriched environments combined with daily training in skilled reaching and gridwalk crossing improve functional recovery of both tasks after a cervical spinal cord injury in rats. A right-sided cervical overhemisection lesion of the spinal cord at C4-5 caused significant impairment of both right and left reaching and hindlimb and forelimb locomotion. Animals recovered some ability to reach with their left forelimbs and to grasp pellets without any additional intervention. Daily training significantly improved reaching on the left side but not on the right side. There were significantly fewer errors made while crossing a gridwalk. Enriched environments and daily training did not improve forelimb function during vertical exploration or locomotion, both automatic behaviors.

FIG. 5. Enriched environments with daily training slightly reduces the hindlimb base of support. (A) Catwalk gait analysis was used to measure base of support, stride length, standtime, and swingtime during locomotion. A two-way ANOVA was used to compare the effects of enriched housing and daily training or rolipram on hindlimb base of support (B) and forelimb base of support (C). Animals housed in enriched environments with daily training had a significant reduction in the hindlimb base of support $(p<0.01)$. Data shown are from 4 weeks after injury and treatment. Values represent the mean \pm SEM. Standard environment: sham, $\mathrm{n}=5 ; \mathrm{HX}, \mathrm{n}=6$; $\mathrm{HX} /$ Veh, $\mathrm{n}=6$; $\mathrm{HX} / \mathrm{Rp}$, $\mathrm{n}=9$. Enriched environment: sham, $\mathrm{n}=5 ; \mathrm{HX}, \mathrm{n}=6$; $\mathrm{HX} /$ Veh, $\mathrm{n}=5 ; \mathrm{HX} / \mathrm{Rp}, \mathrm{n}=10$. Sham, sham-operated rats; HX, cervical over-hemisection of the spinal cord; Veh, vehicle; Rp, rolipram. ${ }^{*} p<0.05$. 


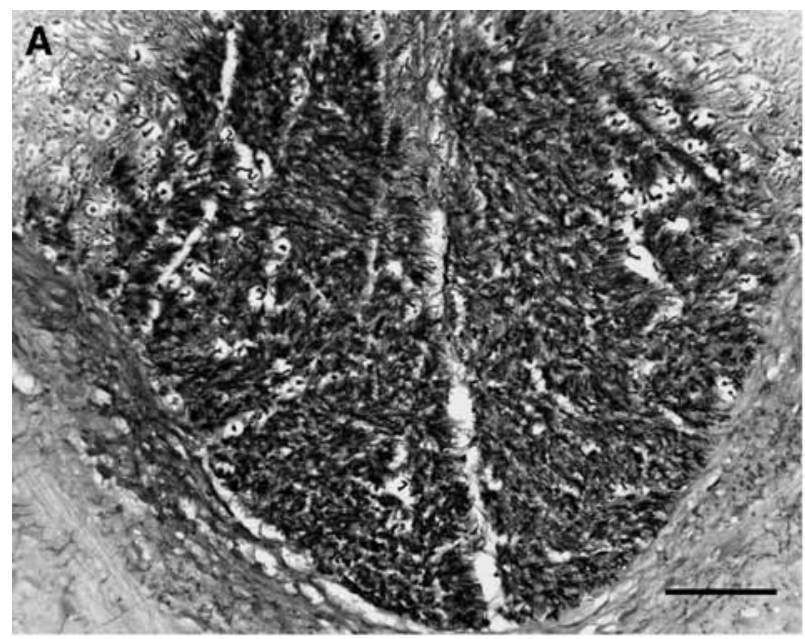

C
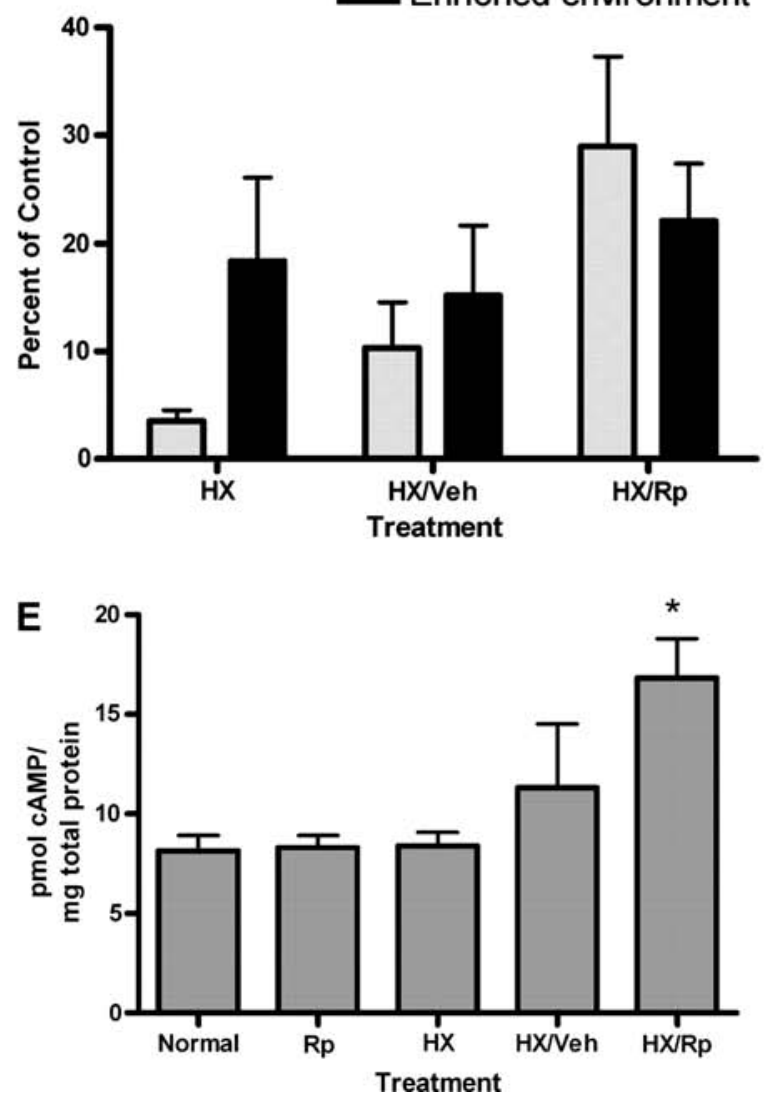

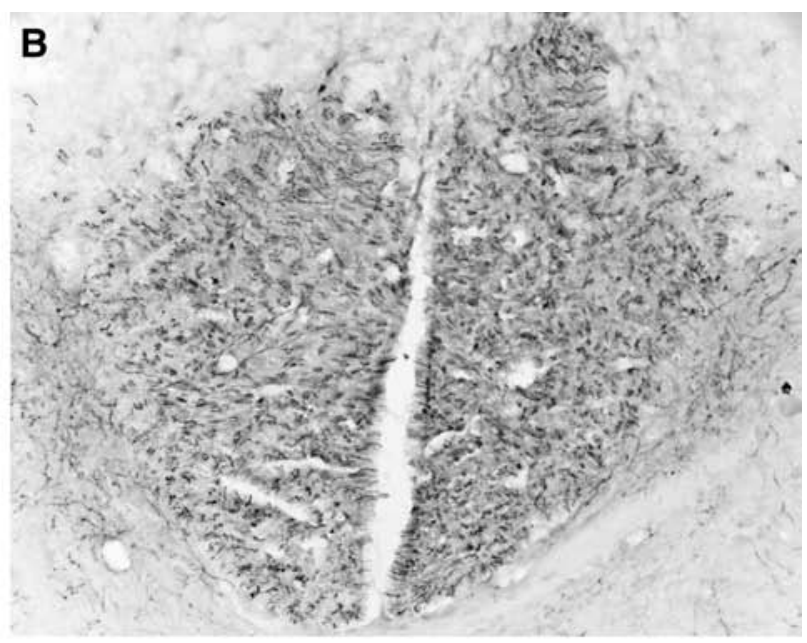

D

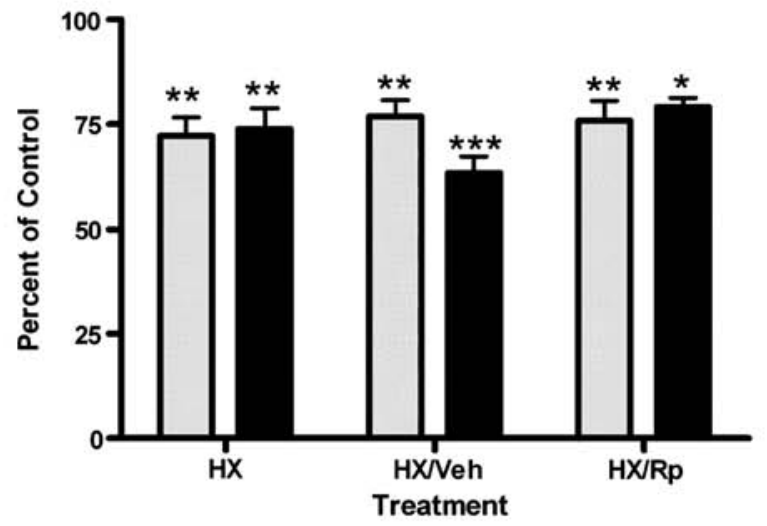

FIG. 6. Both enriched environments with daily training and rolipram promote plasticity of the corticospinal tract rostral to lesion. Corticospinal axons were anterogradely labeled with BDA. Serial cross sections $(20 \mu \mathrm{m})$ located $8 \mathrm{~mm}$ rostral to lesion were incubated with avidin-biotin horseradish peroxidase complex and developed with DAB enhanced with nickel. Photomicrographs of sections from sham-operated animals (A) housed in standard housing and after cervical over-hemisection (B) are shown. (C) Dorsal column labeling rostral to injury. Stained sections were digitally captured and BDA positive fibers were measured in the dorsal column (pixel area). Data were normalized to control rats that received sham surgery and were housed in standard cages. Sham-operated rats housed in enriched housing with daily training gave identical results to those housed in standard housing. A two-way ANOVA was used to compare groups. HX, cervical over-hemisection of the spinal cord; Veh, vehicle; Rp, rolipram. Standard environment: sham $n=5 ; H X, n=7 ; H X / V e h, n=6 ; H X / R p, n=9$. Enriched environment: sham $\mathrm{n}=6, \mathrm{HX}, \mathrm{n}=7, \mathrm{HX} / \mathrm{Veh} \mathrm{n}=6, \mathrm{HX} / \mathrm{Rol} \mathrm{n}=9$. (D) The cross-sectional area of BDA labeled corticospinal tract fibers in the dorsal column was measured and normalized as done for panel C. Analysis was as that for C: ${ }^{*} p<0.05,{ }^{* *} p<0.01,{ }^{* * *} p<0.001$. (E) The effect of rolipram on cAMP content in the spinal cord was measured. Two weeks after injury, rolipram or vehicle were administered for 5 days and the cervical spinal cord was harvested. A one-way ANOVA was performed. Normal, $n=3 ; R p, n=5 ; H X$, $\mathrm{n}=3 ; \mathrm{HX} /$ Veh, $\mathrm{n}=3 ; \mathrm{HX} / \mathrm{Rp}, \mathrm{n}=4$. Values represent mean \pm SEM. ${ }^{*} p<0.05,{ }^{* *} p<0.01,{ }^{* * *} p<0.001$. scale bar for $\mathrm{A}=100 \mu \mathrm{m}$. 
Injury, activity, and plasticity of spared pathways influence the type of anatomical reorganization and motor recovery

After lesion, rats lost right shoulder function and were unable to lift their right forelimbs to reach or place their forepaw against the wall during vertical exploration. As a result, reach training occurred primarily with the left forelimb. This may have further exacerbated the loss of right reaching through learned disuse of the right side (Taub and Uswatt, 2006; Allred et al., 2005) and reduced neuronal activation in the cortex (Allred and Jones, 2007).

Plasticity of spared pathways likely provided a substrate for recovery of left reaching in this study. Reaching requires both proximal forelimb use for shoulder lift and arm extension, and distal forelimb use for digit control to grasp the pellet and retract (Whishaw et al., 1986). Rats with medial lesions of the spinal cord, which remove corticospinal tract axons in the dorsal column, exhibit deficits in pellet retrieval after injury but recover over time. Rats with lateral lesions, which destroy the dorsolateral corticospinal tract and rubrospinal tract, exhibit more permanent deficits (Anderson et al., 2007). In the over-hemisection, the left rubrospinal tract and ventral white matter, areas shown to support recovery of forelimb function, are left intact after lesion (Keyvan-Fouladi et al., 2003; Anderson et al., 2005). Furthermore, substantial recovery of skilled forelimb movements in rats is subserved by structural plasticity of the ventral corticospinal tract (a tract spared by our lesion but not normally responsible for skilled reaching in intact animals). Recovery is correlated with increased contacts between ventral corticospinal tract axons and motorneurons in the cervical spinal cord (Weidner et al., 2001). Thus, daily reach training may promote plasticity of pathways normally involved in reaching, as well as recruit alternate pathways.

Interestingly, training in a skilled task creates a different experience supraspinally than training in an automatic task. Skilled reach training induces reorganization of the motor cortex (Kleim et al., 1998; Adkins et al., 2006), inducing synaptogenesis, synaptic potentiation, and reorganization of the forelimb representation (Jones et al., 1999). In contrast, neither endurance training nor strength training in rats alters motor map organization (Adkins et al., 2006). Furthermore, skilled training can be potentiated by increased activity. Electrical stimulation applied during skilled reach training further improves reaching after lesion of the sensorimotor cortex in rats (Adkins et al., 2006) and stimulation of silenced corticospinal tract axons in kittens partially restores connectivity and significantly improves motor function during the horizontal ladder (Salimi et al., 2008), suggesting both increased activity and skilled training contribute to recovery of skilled tasks. After a unilateral lesion of the pyramidal tract, both injury and electrical stimulation of spared corticospinal tract axons independently strengthened their connectivity, resulting in a novel pattern of terminations within the ventral cord (BrusRamer et al., 2007).

While the mechanisms for recovery on the gridwalk are unclear, gridwalk requires motor control for accurate limb placement integrated with sensory feedback from the grid (Barth et al., 1990; Kunkel-Bagden et al., 1993; Schucht et al., 2002). Locomotion on the gridwalk requires preservation of fibers in the ventrolateral and dorsolateral funiculi (Schucht et al., 2002), areas containing reticulospinal, rubrospinal, and vestibulospinal pathways. These areas were spared unilaterally by the cervical over-hemisection lesion in this study, suggesting plasticity of these spared pathways supported recovery of sensorimotor function. Improved limb function on the gridwalk resulted in reduced foot faults or errors made while traversing the grid runway. Improved right forelimb function on the gridwalk was likely due to compensatory movements involving other limbs, such as shifting weight during locomotion, to provide greater control and cause fewer faults. Additional improvements in sensory function or from learning the task could have further contributed to the observed decrease in errors.

\section{Lack of recovery of automatic forelimb behavior without a tissue graft}

Daily training in skilled reaching and gridwalk did not improve forelimb use during locomotion or vertical exploration. Animals reared up on their hindlimbs after lesion, as reported previously (Lynskey et al., 2006), but rarely used their right limbs for support. Post-injury housing in enriched environments with training slightly reduced the hindlimb base of support, as assessed by CatWalk analysis (Hamers et al., 2001, 2006), suggesting greater stability during locomotion. However, there was no significant improvement in forelimb use during locomotion, and other parameters of locomotion, including increased standtime and reduced stride length after injury, were unaffected by treatment.

Automatic forelimb movements, such as those that occur during vertical exploration or locomotion, are more dependent upon local segmental pathways (Metz et al., 1998; Muir and Whishaw, 1999; Juvin et al., 2005; Piecharka et al., 2005) and are associated with serotonergic reinnervation of the spinal pattern generator by raphespinal axons (Ribotta et al., 2000). Recovery of quadrupedal locomotion in cats after partial spinal cord lesions is facilitated by locomotor training on the treadmill (Barriere et al., 2008), and mostly results from intrinsic changes below the lesion in the central pattern generator and afferent inputs. Interestingly, our recent studies suggest that forelimb use during automatic behaviors may benefit from cellular replacement at the injury site since animals show better right forelimb use during rearing (Nikulina et al., 2004) and locomotion (Lynskey et al., unpublished observations) when fetal spinal cord transplants were grafted at the lesion site. The presence of the graft may have provided a suitable substrate for axon growth as well an opportunity for corticospinal axons to access motor neurons or interneurons that support the recovery of forelimb motor function. Thus, on the right side of the spinal cord, loss of segmental circuitry and the lack of structure to support axon growth conducive to recovering right forelimb use, mean that descending corticospinal and rubrospinal pathways have no right forelimb "targets" with which to form connections.

\section{Rolipram did not improve functional recovery without a tissue graft}

Somewhat surprisingly, rolipram did not improve recovery of skilled or automatic forelimb use in this study. Rolipram selectively inhibits phosphodiesterase 4 (PDE4) in cortical neurons and raises cAMP (Yamashita et al., 1997). Rolipram infusion increased spinal cord cAMP levels after injury, but 
did not affect cAMP levels in the uninjured spinal cord, and while not significant, we observed an increase in corticospinal tract labeling rostral to lesion after rolipram.

Previous studies indicate that rolipram or elevated cAMP generates a more permissive environment for axon growth after injury by inhibiting glial proliferation (Zhang et al., 2002) and scar formation (Nikulina et al., 2004; Klapka et al., 2005). After a cervical spinal cord injury, rolipram improves right paw placement during vertical exploration (Nikulina et al., 2004), and after a contusion of the rat thoracic spinal cord, rolipram and db-cAMP increased raphespinal fiber growth into Schwann cell grafts, promoted sparing of supraspinal and propriospinal axons, and improved locomotor function (Pearse et al., 2004). One difference between our current study and our previous study (Nikulina et al., 2004) is that fetal spinal cord tissue was not grafted to the injury site. Further, recovery of locomotion after a thoracic injury would likely be more dependent on plasticity of segmental pathways whereas recovery of forelimb use after a cervical injury involves greater input from supraspinal pathways and was likely precluded by a lack of restoration of segmental circuitry on the ipsilateral side of the spinal cord.

One caveat to this study is that housing in enriched environments was combined with skilled training so we cannot determine if skilled recovery is due to training alone or training plus enriched environments. However, while a number of studies indicate that enriched environments improve sensory and motor function after spinal cord injury (Lankhorst et al., 2001; Van Meeteren et al., 2003; Koopmans et al., 2006; Berrocal et al., 2007), post-injury enrichment alone does not improve skilled reaching after cerebral ischemia (Grabowski et al., 1993) and recovery of skilled function after CNS injury requires additional rehabilitation with specific skill training to reverse skilled motor deficits (Biernaskie and Corbett, 2001; Girgis et al., 2007; Maldonado et al., 2007). Therefore, in this study we combined both increased activity (enriched environment housing) with daily skilled forelimb training to optimize recovery of skilled function.

\section{Cervical spinal cord injuries and behavioral outcomes}

In this study deficits to the limbs after cervical overhemisection were very similar to those reported after cervical contusion of the spinal cord (Schrimsher and Reier, 1992; Soblosky et al., 2001; Gensel et al., 2006). For both types of injuries, the majority of rats used dorsal forepaw placement of the right forelimb for the first 1-2 weeks after surgery and by 4 weeks, clubbing of the right forepaw was observed in over $50 \%$ of the rats (data not shown). Rats showed reduction in the use of their forelimbs, had significant deficits in the hindlimbs (Soblosky et al., 2001), and were unable to perform pellet retrieval (Schrimsher and Reier, 1992). Rats made significantly more forelimb and hindlimb errors on the horizontal ladder (Gensel et al., 2006), and had a reduced stride length and an increased base of support (Pearse et al., 2005). After unilateral contusions, rats preferred the contralateral limb during vertical exploration (Soblosky et al., 2001; Gensel et al., 2006). With the unilateral contusion injury, while there was greater tissue sparing laterally, there appeared to be a greater lesion volume rostral to caudal compared to surgical over-hemisection. Given the similarities between the behavioral outcomes after cervical contusion and surgical over- hemisection injuries, it will be interesting to see if animals with these injuries respond similarly to daily rehabilitation training.

In summary, training in skilled reaching and gridwalk improves recovery of left forelimb reaching and skilled forelimb function but does not improve automatic forelimb function after a cervical spinal cord injury, suggesting these functions must be rehabilitated independently. Furthermore, multiple skilled functions, such as reaching and gridwalk, can be trained and rehabilitated together. Recovery is associated with plasticity of the rostral corticospinal tract and is likely to be dependent on plasticity of spared pathways. It remains to be determined whether multiple automatic functions can be recovered concurrently with multiple skilled functions.

\section{Acknowledgments}

Thanks to Drs. Francoise Seillier-Moiseiwitsch and Huwaida Rabie from the Department of Biostatistics, Bioinformatics, and Biomathematics at Georgetown University Medical Center and to Paramita Das for her help with data analysis. Thanks to Dr. Nathan Neckel for a critical review of the manuscript. This work was supported by the Christopher and Dana Reeve Paralysis Foundation (BA2-0412-2) and by a grant from the NIH/NINDS (NS 051656, to BSB).

\section{Author Disclosure Statement}

CatWalk was developed by Dr. Hamers during his tenure as assistant professor at the Department of Pharmacology and Anatomy, Rudolf Magnus Institute of Neuroscience, University Medical Center Utrecht. CatWalk is now commercialized by Noldus Information Technology B.V., Wageningen, The Netherlands. Both the Department of Pharmacology and Anatomy and Dr. Hamers receive some financial benefit from this commercialization.

\section{References}

Adkins, D.L., Boychuk, J., Remple, M.S., and Kleim, J.A. (2006). Motor training induces experience-specific patterns of plasticity across motor cortex and spinal cord. J. Appl. Physiol. 101, 1776-1782.

Adkins, D.L., Campos, P., Quach, D., Borromeo, M., Schallert, K., and Jones, T.A. (2006). Epidural cortical stimulation enhances motor function after sensorimotor cortical infarcts in rats. Exp. Neurol. 200, 356-370.

Allred, R.P., and Jones, T.A. (2008). Maladaptive effects of learning with the less-affected forelimb after focal cortical infarcts in rats. Exp. Neurol. 210, 172-186.

Allred, R.P., Maldonado, M.A., Hsu, J.E., and Jones, T.A. (2005). Training the "less-affected" forelimb after unilateral cortical infarcts interferes with functional recovery of the impaired forelimb in rats. Restor. Neurol. Neurosci. 23, 297-302.

Alstermark, B., and Kummel, H. (1986). Transneuronal labelling of neurones projecting to forelimb motoneurons in cat performing different movements. Brain Res. 376, 387-391.

Alstermark, B., Kummel, H., Pinter, M., and Tantisira, B. (1987). Branching and termination of $\mathrm{C} 3-\mathrm{C} 4$ propriospinal neurones in the cervical spinal cord of the cat. Neurosci. Lett. 74, 291296.

Alstermark, B., Kummel, H., Pinter, M.J., and Tantisira, B. (1990). Integration in descending motor pathways controlling the 
forelimb in the cat. 17. Axonal projection and termination of C3-C4 propriospinal neurones in the C6-Th1 segments. Exp. Brain Res. 81, 447-461.

Anderson, K.D., Gunawan, A., and Steward, O. (2005). Quantitative assessment of forelimb motor function after cervical spinal cord injury in rats: relationship to the corticospinal tract. Exp. Neurol. 194, 161-174.

Anderson, K.D., Gunawan, A., and Steward, O. (2007). Spinal pathways involved in the control of forelimb motor function in rats. Exp. Neurol. 206, 318-331.

Barriere, G., Leblond, H., Provencher, J., and Rossignol, S. (2008). Prominent role of the spinal central pattern generator in the recovery of locomotion after partial spinal cord injuries. J. Neurosci. 28, 3976-3987.

Barth, T.M., Jones, T.A., and Schallert, T. (1990). Functional subdivisions of the rat somatic sensorimotor cortex. Behav. Brain Res. 39, 73-95.

Berrocal, Y., Pearse, D.D., Singh, A., Andrade, C.M., McBroom, J.S., Puentes, R., and Eaton, M.J. (2007). Social and environmental enrichment improves sensory and motor recovery after severe contusive spinal cord injury in the rat. J. Neurotrauma 24, 1761-1772.

Biernaskie, J., and Corbett, D. (2001). Enriched rehabilitative training promotes improved forelimb motor function and enhanced dendritic growth after focal ischemic injury. J. Neurosci. 21, 5272-5280.

Bregman, B.S., Kunkel-Bagden, E., Reier, P.J., Dai, H., McAtee, M., and Gao, D. (1993). Recovery of function after spinal cord injury: mechanisms underlying transplant-mediated recovery of function differ after spinal cord injury in newborn and adult rats. Exp. Neurol. 123, 3-16.

Brus-Ramer, M., Carmel, J.B., Chakrabarty, S., and Martin, J.H. (2007). Electrical stimulation of spared corticospinal axons augments connections with ipsilateral spinal motor circuits after injury. J. Neurosci. 27, 13793-13801.

Castro, A.J. (1972). Motor performance in rats. The effects of pyramidal tract section. Brain Res. 44, 313-323.

Diener, P.S., and Bregman, B.S. (1998). Fetal spinal cord transplants support the development of target reaching and coordinated postural adjustments after neonatal cervical spinal cord injury. J. Neurosci. 18, 763-778.

Engesser-Cesar, C., Anderson, A.J., Basso, D.M., Edgerton, V.R., and Cotman, C.W. (2005). Voluntary wheel running improves recovery from a moderate spinal cord injury. J. Neurotrauma 22, 157-171.

Gensel, J.C., Tovar, C.A., Hamers, F.P., Deibert, R.J., Beattie, M.S., and Bresnahan, J.C. (2006). Behavioral and histological characterization of unilateral cervical spinal cord contusion injury in rats. J. Neurotrauma 23, 36-54.

Girgis, J., Merrett, D., Kirkland, S., Metz, G.A.S., Verge, V., and Fouad, K. (2007). Reaching training in rats with spinal cord injury promotes plasticity and task specific recovery. Brain 130, 2993-3003.

Grabowski, M., Brundin, P., and Johansson, B.B. (1993). Pawreaching, sensorimotor, and rotational behavior after brain infarction in rats. Stroke 24, 889-895.

Hamers, F.P.T., Koopmans, G.C., and Joosten, E.A.J. (2006). Catwalk-assisted gait analysis in the assessment of spinal cord injury. J. Neurotrauma 23, 537-548.

Hamers, F.P.T., Lankhorst, A.J., Van Laar, T.J., Veldhuis, W.B., and Gispen, W.H. (2001). Automated quantitative gait analysis during overground locomotion in the rat: its application to spinal cord contusion and transection injuries. J. Neurotrauma $18,187-201$.
Hutchinson, K.J.K., Gomez-Pinilla, F., Crowe, M.J., Ying, Z., and Basso, D.M. (2004). Three exercise paradigms differentially improve sensory recovery after spinal cord contusion in rats. Brain 127, 1403-1414.

Johansson, B.B. (1996). Functional outcome in rats transferred to an enriched environment 15 days after focal brain ischemia. Stroke 27, 324-326.

Jones, T.A., Chu, C.J., Grande, L.A., and Gregory, A.D. (1999). Motor skills training enhances lesion-induced structural plasticity in the motor cortex of adult rats. J. Neurosci. 19, 1015310163.

Juvin, L., Simmers, J., and Morin, D. (2005). Propriospinal circuitry underlying interlimb coordination in mammalian quadrupedal locomotion. J. Neurosci. 25, 6025-6035.

Keyvan-Fouladi, N., Raisman, G., and Li, Y. (2003). Functional repair of the corticospinal tract by delayed transplantation of olfactory ensheathing cells in adult rats. J. Neurosci. 23(28), 9428-9434.

Klapka, N., Hermanns, S., Straten, G., Masanneck, C., Duis, S., Hamers, F.P.T., Muller, D., Zuschratter, W., and Muller, H.W. (2005). Suppression of fibrous scarring in spinal cord injury of rat promotes long-distance regeneration of corticospinal tract axons, rescue of primary motoneurons in somatosensory cortex and significant functional recovery. Eur. J. Neurosci. 22, 3047-3058.

Kleim, J.A., Barbay, S., and Nudo, R.J. (1998). Functional reorganization of the rat motor cortex following motor skill learning. J. Neurophysiol. 80, 3321-3325.

Komitova, M., Zhao, L.R., Gido, G., Johansson, B.B., and Eriksson, P. (2005). Postischemic exercise attenuates whereas enriched environment has certain enhancing effects on lesioninduced subventricular zone activation in the adult rat. Eur. J. Neurosci. 21, 2397-2405.

Koopmans, G.C., Brans, M., Gomez-Pinella, F., Duis, S., Gispen, W.H., Torres-Aleman, I., Joosten, E.A.J., and Hamers, F.P.T. (2006). Circulating insulin-like growth factor 1 and functional recovery from spinal cord injury under experimental housing conditions. Eur. J. Neurosci. 23, 1035-1046.

Krause, W., and Kuhne, G. (1988). Pharmacokinetics of rolipram in the rhesus and cynomolgus monkeys, the rat and the rabbit. Studies on species differences. Xenobiotica 18, 561-571.

Kunkel-Bagden, E., Dai, H.-N., and Bregman, B.S. (1993). Methods to assess the development and recovery of locomotor function after spinal cord injury in rats. Exp. Neurol. 119, 153-164.

Lankhorst, A.J., ter Laak, M.P., van Laar, T.J., van Meeteren, N.L.U., de Groot, J.C.M.J., Schrama, L.H., Hamers, F.P.T., and Gispen, W.H. (2001). Effects of enriched housing on functional recovery after spinal cord contusive injury in the adult rat. J. Neurotrauma 18, 203-215.

Lynskey, J.V., Sandhu, F.A., Dai, H.N., McAtee, M., Slotkin, J.R., MacArthur, L., and Bregman, B.S. (2006). Delayed intervention with transplants and neurotrophic factors supports recovery of forelimb function after cervical spinal cord injury in adult rats. J. Neurotrauma 23, 617-634.

Maegele, M., Lippert-Gruener, M., Ester-Bode, T., Garbe, J., Bouillon, B., Neugebauer, E., Klug, N., Lefering, R., Neiss, W.F., and Angelov, D.N. (2005). Multimodal early onset stimulation combined with enriched environment is associated with reduced CNS lesion volume and enhanced reversal of neuromotor dysfunction after traumatic brain injury in rats. Eur. J. Neurosci. 21, 2406-2418.

Maldonado, M.A., Allred, R.P., Felthauser, E.L., and Jones, T.A. (2007). Motor skill training, but not voluntary exercise, improves recovery of skilled reaching after unilateral ischemic 
sensorimotor cortex lesions in rats. Neurorahabil. Neural Repair 22, 250-261.

Marin, R., Williams, A., Hale, S., Burge, B., Mense, M., Bauman, R., and Tortella, F. (2003). The effect of voluntary exercise exposure on histological and neurobehavioral outcomes after ischemic brain injury in the rat. Physiol. Behav. 80, 167-175.

Metz, G.A., Dietz, V., Schwab, M.E., and van de Meent, H. (1998). The effects of unilateral pyramidal tract section on hindlimb motor performance in the rat. Behav. Brain Res. 96, 37-46.

Muir, G.D., and Whishaw, I.Q. (1999). Complete locomotor recovery following corticospinal tract lesions: measurement of ground forces during overground locomotion in rats. Behav. Brain Res. 103, 45-53.

Nikulina, E., Tidwell, J.L., Dai, H.N., Bregman, B.S., and Filbin, M.T. (2004). The phosphodiesterase inhibitor rolipram delivered after a spinal cord lesion promotes axonal regeneration and functional recovery. Proc.Natl. Acad. Sci. 101, 8786-8790.

Nithianantharajah, J., and Hannan, A.J. (2006). Enriched environments, experience-dependent plasticity and disorders of the nervous system. Nat. Rev. Neurosci. 7, 697-709.

Ohlsson, A.L., and Johansson, B.B. (1995). Environment influences functional outcome of cerebral infarction in rats. Stroke 26, 644-649.

Pearse, D.D., Lo Jr., T.P., Cho, K.S., Lynch, M.P., Garg, M.S., Marcillo, A.E., Sanchez, A.R., Cruz, Y., and Dietrich, W.D. (2005). Histopathological and behavioral characterization of a novel cervical spinal cord displacement contusion injury in the rat. J. Neurotrauma 22, 680-702.

Pearse, D.D., Pereira, F.A., Marcillo, A.E., Bates, M.L., Berrocal, Y.A., Filbin, M.T., and Bunge, M.B. (2004). cAMP and Schwann cells promote axonal growth and functional recovery after spinal cord injury. Nat. Med. 10, 610-616.

Piecharka, D.M., Kleim, J.A., and Whishaw, I.Q. (2005). Limits on recovery in the corticospinal tract of the rat: partial lesions impair skilled reaching and the topographic representation of the forelimb in motor cortex. Brain Res. Bull. 66, 203-211.

Ploughman, M., Attwood, Z., White, N., Dore, J.J., and Corbett, D. (2007). Endurance exercise facilitates relearning of forelimb motor skill after focal ischemia. Eur. J. Neurosci. 25, 3453-3460.

Reiner, A., Veenman, C.L., Medina, L., Jiao, Y., Del Mar, N., and Honig, M.G. (2000). Pathway tracing using biotinylated dextran amines. J. Neurosci. Methods 103, 23-37.

Ribotta, M., Provencher, J., Feraboli-Lohnher, D., Rossignol, S., Privat, A., and Orsal, D. (2000). Activation of locomotion in adult chronic spinal rats is achieved by transplantation of embryonic raphe cells reinnervating a precise lumbar level. J. Neurosci. 20, 5144-5152.

Risedal, A., Mattsson, B., Dahlqvist, P., Nordborg, C., Olsson, T., and Johansson, B.B. (2002). Environmental influences on functional outcome after a cortical infarct in the rat. Brain Res. Bull. $58,315-321$.

Salimi, I., Friel, K.M., and Martin, J.H. (2008). Pyramidal tract stimulation restores normal corticospinal tract connections and visuomotor skill after early postnatal motor cortex activity blockade. J. Neurosci. 28, 7426-7434.

Schallert, T., Fleming, S.M., Leasure, J.L., Tillerson, J.L., and Bland, S.T. (2000). CNS plasticity and assessment of forelimb sensorimotor outcome in unilateral rat models of stroke, cortical ablation, parkinsonism and spinal cord injury. Neuropharmacology 39, 777-787.

Schrimsher, G.W., and Reier, P. (1992). Forelimb motor performance following cervical spinal cord contusion injury in the rat. Exp. Neurol. 117, 287-298.

Schucht, P., Raineteau, O., Schwab, M.E., and Fouad, K. (2002). Anatomical correlates of locomotor recovery following dorsal and ventral lesions of the rat spinal cord. Exp. Neurol. 176, 143-153.

Soblosky, J.S., Song, J.-H., and Dinh, D.H. (2001). Graded unilateral cervical spinal cord injury in the rat: evaluation of forelimb recovery and histological effects. Behav. Brain Res. 119, 1-13.

Taub, E., and Uswatt, G. (2006). Constraint-induced movement therapy: answers and questions after two decades of research. NeuroRehabilitation 21, 93-95.

Van Meeteren, N.L., Eggers, R., Lankhorst, A.J., Gispen, W.H., and Hamers, F.P.T. (2003). Locomotor recovery after spinal cord contusion injury in rats is improved by spontaneous exercise. J. Neurotrauma 20, 1029-1037.

van Praag, H., Shubert, T., Zhao, C., and Gage, F.H. (2005). Exercise enhances learning and hippocampal neurogenesis in aged mice. J. Neurosci. 25, 8680-8685.

Weidner, N., Ner, A., Salimi, N., and Tuszynski, M.H. (2001). Spontaneous corticospinal axonal plasticity and functional recovery after adult central nervous system injury. Proc. Natl. Acad. Sci. 98, 3513-3518.

Whishaw, I.Q., Gorny, B., and Sarna, J. (1998). Paw and limb use in skilled and spontaneous reaching after pyramidal tract, red nucleus and combined lesions in the rat: behavioral and anatomical dissociations. Behav. Brain Res. 93, 167-183.

Whishaw, I.Q., O'Connor, W.T., and Dunnett, S.B. (1986). The contributions of motor cortex, nigrostriatal dopamine and caudate-putamen to skilled forelimb use in the rat. Brain 109, 805-843.

Yamashita, N., Yamauchi, M., Baba, J., and Sawa, A. (1997). Phosphodiesterase type 4 that regulates cAMP level in cortical neurons shows high sensitivity to rolipram. Eur. J. Pharmacol. 337, 95-102.

Zhang, B., Yang, L., Konishi, Y., Maeda, N., Sakanaka, M., and Tanaka, J. (2002). Suppressive effects of phosphodiesterase type IV inhibitors on rat cultured microglial cells: comparison with other types of cAMP-elevating agents. Neuropharmacology 42, 262-269.

Address correspondence to: Barbara S. Bregman, M.D.

Department of Neuroscience Georgetown University Medical Center NRB Rm. EP-04 3970 Reservoir Road NW Washington, DC 20007

E-mail: bregmanb@georgetown.edu 